\title{
A Continuous Approach for the Computation of the Hyperbolic Singular Value Decomposition
}

\author{
T. Politi \\ Dipartimento di Matematica, Politecnico di Bari, \\ Via Amendola 126/B, I-70126 Bari (Italy). \\ politi@poliba.it
}

\begin{abstract}
In this paper a continuous approach based on the Projected Gradient Flow technique is presented in order to find a generalization of the Singular Value Decomposition (SVD) of a rectangular matrix called Hyperbolic SVD. If $A$ is a $m \times n$ real matrix with full column rank and if $G$ is a $n \times n$ diagonal sign matrix, i.e. $g_{i i}= \pm 1$, the Hyperbolic Singular Value Decomposition of the pair $(A, G)$ is defined as $A=U \Sigma V$, where $U$ is orthogonal, $\Sigma$ is diagonal with positive entries and $V$ is hypernormal (or $G$-orthogonal), i.e. $V^{T} G V=G$. In this work we use a continuous approach based on the projected gradient technique obtaining two differential systems, the first one evolving on group of orthogonal matrices and the second on the quadratic group related to $G$. A numerical test is reported in order to show the effectiveness of the approach.
\end{abstract}

\section{Introduction}

Recently there has been a growing interest in numerical analysis community in the exploitation of the concept of structure associated to matrices. Examples of these structures are orthogonality, simplecticity, skew-symmetry and so on. Some of these properties are related to group structures (and sometimes to Lie-group structures) or to algebra structure (for example the skew-symmetry) or, more simplicity to algebraic properties (it is the case of the obliqueness, i.e. matrices $Y$ such that $\left.\operatorname{diag}\left(Y^{T} Y\right)=I\right)$. In particular a great attention has been devoted to the numerical solution of differential equations evolving on matrix groups (see [7] for quadratic groups, 6] for Lie groups, [3,4] for orthogonal matrices). The experience in the effective solution of differential equations on matrix groups can be used also to solve some linear algebra problems, such as the computation of factorizations of time depending matrices (see 13]) or the computation of singular value decomposition factors as limit point of continuous flows (see [2]). In this paper we consider this last problem in the case of the Hyperbolic Singular Value Decomposition. The work is organized as follows: in Section 2 we define the Hyperbolic Singular Value Decomposition and recall some important features and applications, in Section 3 we use the projected gradient technique in order to obtain two differential flows having, respectively, the unitary and the hypernormal factors as limit point. Finally in the Section 4 a numerical test is described in order to show the effectiveness of the differential approach. 


\section{The Hyperbolic Singular Value Decomposition}

In this section we define the Hyperbolic SVD of a $m \times n$ real matrix $A$ with a couple of its applications, but first we give some important definitions.

Definition 1. Let $G$ be a $m \times m$ diagonal matrix with entries \pm 1 , then a matrix $U \in \mathbb{R}^{m \times m}$ is said hypernormal if $U^{T} G U=G$.

In [5] the hypernormal matrices are called $G$-orthogonal.

Definition 2. If $G$ is a $m \times m$ diagonal matrix with entries \pm 1 , then a matrix $U \in \mathbb{R}^{m \times m}$ is said hyperexchange if

$$
U^{T} G U=\widehat{G}
$$

where $\widehat{G}$ is another real diagonal matrix with entries \pm 1 .

It is easy to observe that there is a strict relation between hypernormal and hyperexchange matrices. In fact if $V$ is an hyperexchange matrix there exists a permutation matrix $P$ such that $W=V P$ is hypernormal. In fact, from (1) since $G$ and $\widehat{G}$ have the same inertia (i.e. the same number of +1 and -1 ) and there exists a permutation matrix $P$ such that $P G P^{T}=\widehat{G}$, hence

$$
V^{T} G V=\widehat{G}=P G P^{T} \quad \Rightarrow \quad(V P)^{T} G(V P)=G
$$

and $W=V P$ is hypernormal. Considering the quadratic group related to matrix $G$, i.e. the set

$$
\mathcal{H}_{G}=\left\{Y \in \mathbb{R}^{n \times n} \mid \operatorname{det}(Y) \neq 0, Y G Y^{T}=G\right\}
$$

we observe that it coincides with the set of hypernormal matrices. Moreover we shall denote by $\mathcal{O}(n)$ as the set of orthogonal matrices of order $n$. The following result states the existence of the Hyperbolic SVD (see [10]).

Theorem 1. Let $A \in \mathbb{R}^{m \times n}$ and $G$ be a square diagonal real matrix of order $n$ with entries equal to \pm 1 , if the rank of $A G A^{T}$ is equal to $\min \{m, n\}$ then there exist a $m \times m$ unitary matrix $U$, an hypernormal $n \times n$ matrix $V$ and $a m \times n$ diagonal matrix $\Sigma$ with positive entries such that

$$
A=U \Sigma V
$$

We observe that in 18 the matrix $V$ is requested to be an hyperexchange matrix but we have already shown that the two sets of matrices are strictly related. The Hyperbolic SVD has some interesting applications: for example in 12 it has been used to solve the symmetric indefinite eigenvalues problem $A \mathbf{x}=\lambda \mathbf{x}$, where $A$ is a square matrix. The algorithm proposed consists of two steps:

- $A$ is decomposed by symmetric indefinite factorization $A=L G L^{T}$ (see [9]), being $G$ a diagonal matrix with entries equal to \pm 1 ; 
- the second step is the computation of the Hyperbolic SVD of $L$, i.e. $L=U \Sigma V$. Since

$$
A=L G L^{T}=U \Sigma V G V^{T} \Sigma U^{T}=U G \Sigma^{2} U^{T},
$$

the eigenvalues of $A$ are $\lambda_{i}=g_{i i} \sigma_{i}^{2}$, while the columns of $U$ are the corresponding eigenvectors. If $G=\operatorname{diag}\left(I_{k},-I_{m-k}\right)$ and we divide the matrix $A$ in block form $A=\left(A_{1} A_{2}\right)$, with $A_{1} \in \mathbb{R}^{m \times k}$ and $A_{2} \in \mathbb{R}^{m \times(m-k)}$, then the Hyperbolic $\mathrm{SVD}$ could be used to find the eigenvalues of the matrix

$$
H=A G A^{T}=A_{1} A_{1}^{T}-A_{2} A_{2}^{T}=U G \Sigma^{2} U^{T}
$$

without forming explicitly the matrix.

\section{A Gradient Flow Approach}

The aim of this section is to use the projected gradient in order to construct a continuous steepest descent flow on the manifold $\mathcal{O}(m) \times \mathcal{H}_{G}$. To apply this approach it is necessary to transform the problem into a minimization one with manifold constraints. From (2) it is

$$
\Sigma=U^{-1} A V^{-1}=U^{T} A G V^{T} G .
$$

Since $\Sigma$ must be diagonal it is natural to minimize the distance between the matrix $U^{T} A G V^{T} G$ and the manifold of real diagonal matrices and to choose as projection the operator giving the main diagonal of the matrix. Defining the function

$$
F(U, V)=\left\|U^{T} A G V^{T} G-\operatorname{diag}\left(U^{T} A G V^{T} G\right)\right\|_{F}^{2}
$$

where $U \in \mathcal{O}(m)$ and $V \in \mathcal{H}_{G}$ and, following the same approach as in [2] for the usual SVD, we have to solve the constrained problem:

$$
\begin{array}{ll}
\text { Minimize } & F(U, V)=\left\langle U^{T} A G V^{T} G-P(A), U^{T} A G V^{T} G-P(A)\right\rangle \\
\text { subject to } & U^{T} U=I_{n}, \quad V^{T} G V=G
\end{array}
$$

where $P(A)=\operatorname{diag}\left(U^{T} A G V^{T} G\right)$, and $\langle A, B\rangle$ denotes the Frobenius inner product of two matrices $A, B \in \mathbb{R}^{m \times n}$ defined as

$$
\langle A, B\rangle=\operatorname{trace}\left(A B^{T}\right)=\sum_{i, j} a_{i j} b_{i j} .
$$

As seen in the previous section the set of hypernormal matrices is the quadratic group related to matrix $G$, that is a particular Lie group and having as Lie algebra the set

$$
\mathfrak{h}_{G}=\left\{A \in \mathbb{R}^{n \times n} \mid A G+G A^{T}=0\right\} .
$$

We observe that if $G$ is the identity matrix of order $n$ then $\mathfrak{h}_{G}$ is the set of real skew-symmetric matrices. A property of the Lie algebra is that it is the tangent 
space of $\mathcal{H}_{G}$ at any hypernormal matrix $Q$ is defined as $\mathcal{T}_{Q} \mathcal{H}_{G}=Q \mathfrak{h}_{G}$. If $K \in \mathfrak{h}_{G}$ and $S$ is a $G$-self adjoint matrix (i.e. $S=G S^{T} G$ ) it is

$$
\operatorname{trace}(S K)=0
$$

then $S$ is orthogonal to any $K$ with respect to metric (4). Then the normal space $\mathcal{N}_{Q} \mathcal{H}_{G}$ of $\mathcal{H}_{G}$ at any $Q$, hypernormal matrix, can be expressed as $\mathcal{N}_{Q} \mathcal{H}_{G}=Q \mathcal{S}_{G}$, where $\mathcal{S}_{G}$ is the set of $G$-self adjoint matrices. Function $F(U, V)$ is defined on the Cartesian product $\mathcal{O}(m) \times \mathcal{H}_{G}$. Taking $H \in \mathbb{R}^{m \times m}$ and $K \in \mathbb{R}^{n \times n}$, the Fréchet derivative of $F$ acting on $(H, K) \in \mathbb{R}^{m \times m} \times \mathbb{R}^{n \times n}$ can be considered as

$$
F^{\prime}(U, V) \cdot(H, K)=\frac{\partial F}{\partial U} \cdot H+\frac{\partial F}{\partial V} \cdot K,
$$

where $\Lambda . \eta$ denotes the result of the action by the linear operator $\Lambda$ on $\eta$. We now calculate each action in (5) as follows. First

$$
\begin{aligned}
\frac{\partial F}{\partial V} . K= & \left\langle U^{T} A G K^{T} G-\operatorname{diag}\left(U^{T} A G K^{T} G\right), U^{T} A G V^{T} G-\operatorname{diag}\left(U^{T} A G V^{T} G\right)\right\rangle= \\
= & \left\langle U^{T} A G K^{T} G, U^{T} A G V^{T} G-\operatorname{diag}\left(U^{T} A G V^{T} G\right)\right\rangle+ \\
& -\left\langle\operatorname{diag}\left(U^{T} A G K^{T} G\right), U^{T} A G V^{T} G-\operatorname{diag}\left(U^{T} A G V^{T} G\right)\right\rangle= \\
= & \left\langle U^{T} A G K^{T} G, U^{T} A G V^{T} G-\operatorname{diag}\left(U^{T} A G V^{T} G\right)\right\rangle= \\
= & \left\langle U^{T} A G K^{T}, U^{T} A G V^{T}-\operatorname{diag}\left(U^{T} A G V^{T}\right)\right\rangle= \\
= & \left\langle V G A^{T} U-\operatorname{diag}\left(V G A^{T} U\right), K G A^{T} U\right\rangle= \\
= & \left\langle\left(V G A^{T} U-\operatorname{diag}\left(V G A^{T} U\right)\right) U^{T} A G, K\right\rangle .
\end{aligned}
$$

It follows, from the Riesz representation theorem, that the partial gradient with respect to the Frobenius inner product can be represented as

$$
\frac{\partial F}{\partial V}=\left(V G A^{T} U-\operatorname{diag}\left(V G A^{T} U\right)\right) U^{T} A G .
$$

Then

$$
\begin{aligned}
\frac{\partial F}{\partial U} . H & =\left\langle H^{T} A G V^{T} G-\operatorname{diag}\left(H^{T} A G V^{T} G\right), U^{T} A G V^{T} G-\operatorname{diag}\left(U^{T} A G V^{T} G\right)\right\rangle= \\
& =\left\langle H^{T} A G V^{T} G, U^{T} A G V^{T} G-\operatorname{diag}\left(U^{T} A G V^{T} G\right)\right\rangle= \\
& =\left\langle H^{T} A G V^{T}, U^{T} A G V^{T}-\operatorname{diag}\left(U^{T} A G V^{T}\right)\right\rangle= \\
& =\left\langle H^{T},\left(U^{T} A G V^{T}-\operatorname{diag}\left(U^{T} A G V^{T}\right)\right) V G A^{T}\right\rangle= \\
& =\left\langle A G V^{T}\left(V G A^{T} U-\operatorname{diag}\left(V G A^{T} U\right)\right), H\right\rangle
\end{aligned}
$$

and

$$
\frac{\partial F}{\partial U}=A G V^{T}\left(V G A^{T} U-\operatorname{diag}\left(V G A^{T} U\right)\right) .
$$


The gradient $\nabla F(U, V)$ now must be projected in the Cartesian product of the tangent spaces of the two manifolds, i.e. $\mathcal{T}_{Q} \mathcal{O}(m) \times \mathcal{T}_{Q} \mathcal{H}_{G}$. The tangent space $\mathcal{T}_{Q} \mathcal{O}(m)$ is $Q \mathfrak{h}$, where $\mathfrak{h}$ is the set of real skew-symmetric matrices of order $m$. It is well known that any general matrix $X \in \mathbb{R}^{n \times n}$ can be uniquely splitted as

$$
X=Q \frac{Q^{T} X+X^{T} Q}{2}+Q \frac{Q^{T} X-X^{T} Q}{2}
$$

then the projection $\mathcal{P}_{\mathcal{O}(m)}(X)$ onto the tangent space $\mathcal{T}_{Q} \mathcal{O}(m)$ is given by

$$
\mathcal{P}_{\mathcal{O}(m)}(X)=Q \frac{Q^{T} X-X^{T} Q}{2} .
$$

Similarly it is easy to verify that any matrix $X \in \mathbb{R}^{n \times n}$ has a unique splitting

$$
X=Q\left[\frac{1}{2}\left(G Q^{T} G X-G X^{T} G Q\right)+\frac{1}{2}\left(G Q^{T} G X+G X^{T} G Q\right)\right]
$$

where $Q \in \mathcal{H}_{G}, G Q^{T} G X-G X^{T} G Q \in \mathfrak{h}_{G}$ and $G Q^{T} G X+G X^{T} G Q \in \mathcal{S}_{G}$. The projection of the gradient of $F(U, V)$ into the tangent space $\mathcal{T}_{Q} \mathcal{H}_{G}$ is

$$
\mathcal{P}_{\mathcal{H}_{G}}(X)=Q \frac{G Q^{T} G X-G X^{T} G Q}{2} .
$$

Hence the differential systems that must be solved are

$$
\begin{aligned}
\frac{d U}{d t} & =-\mathcal{P}_{\mathcal{O}(m)}\left(\frac{\partial F}{\partial U}\right) \\
\frac{d V}{d t} & =-\mathcal{P}_{\mathcal{H}_{G}}\left(\frac{\partial F}{\partial V}\right) .
\end{aligned}
$$

\section{Numerical Tests}

In this section we show a numerical example obtained applying the projected gradient flow approach described previously. We consider a $5 \times 3$ real random matrix $A$ having as main diagonal of $\Sigma$ the vector $(0.5,1,1.5)$, and taking $G=\operatorname{diag}\left(I_{2},-1\right)$. The differential systems (6)-(7) have been solved numerically in the interval $[0,30]$. In Figure 1 we show the behaviour of the objective function (3), while in Figure 2 we show the behaviour of the diagonal entries of the matrix $X_{n}=U_{n}^{T} A G V_{n}^{T} G$. Matrices $U_{n}$ and $V_{n}$ are the numerical approximations of the solutions $U(t)$ and $V(t)$, computed at $t=t_{n}$, obtained first integrating the differential systems (6)-(7) with the MatLab ode routine ode113 and then projecting the numerical solutions on the manifolds. For the orthogonal flow (6) the projection has been computed taking the orthogonal factor of the $Q R$ decomposition (see [3] for more details), while for the flow (7) the hypernormal factor of hyperbolic $Q R$ decomposition has been taken (see [11]). In [3] has been 


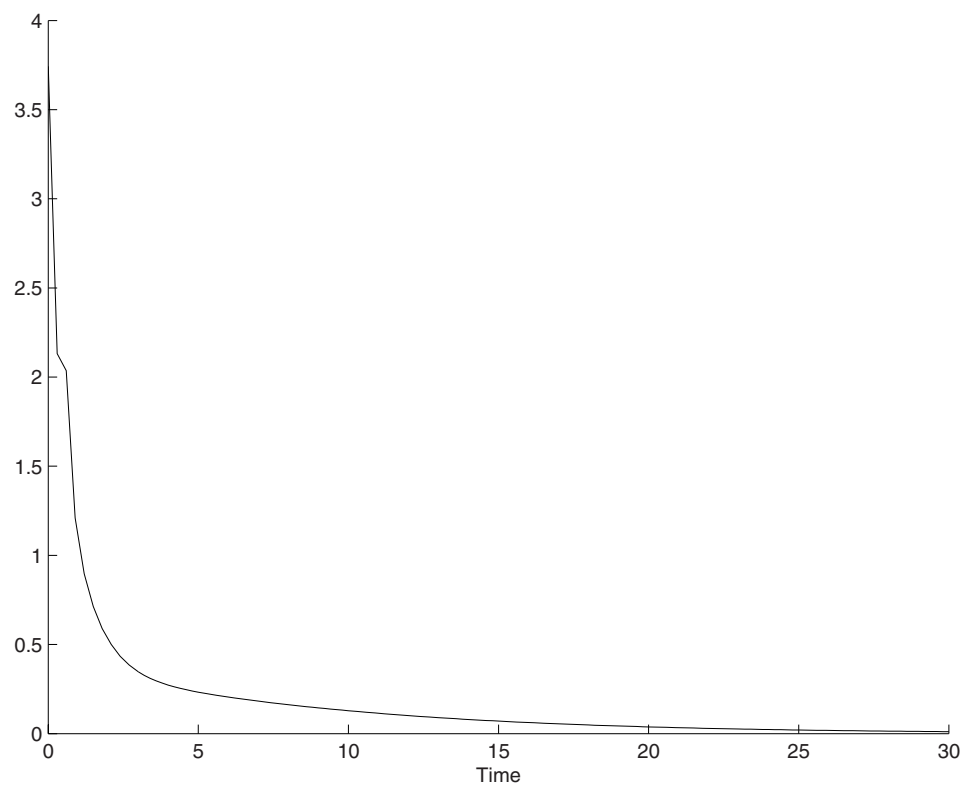

Fig. 1. Evolution of the objective function.

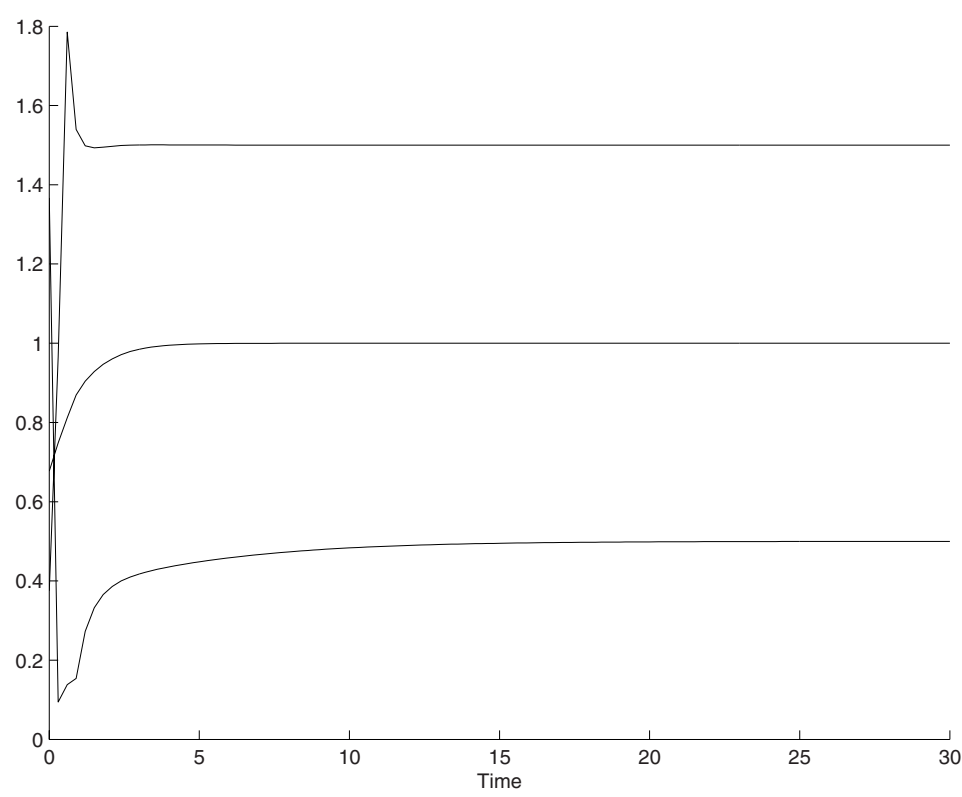

Fig. 2. Diagonal elements of matrices $X_{n}$. 
A Continuous Approach

473
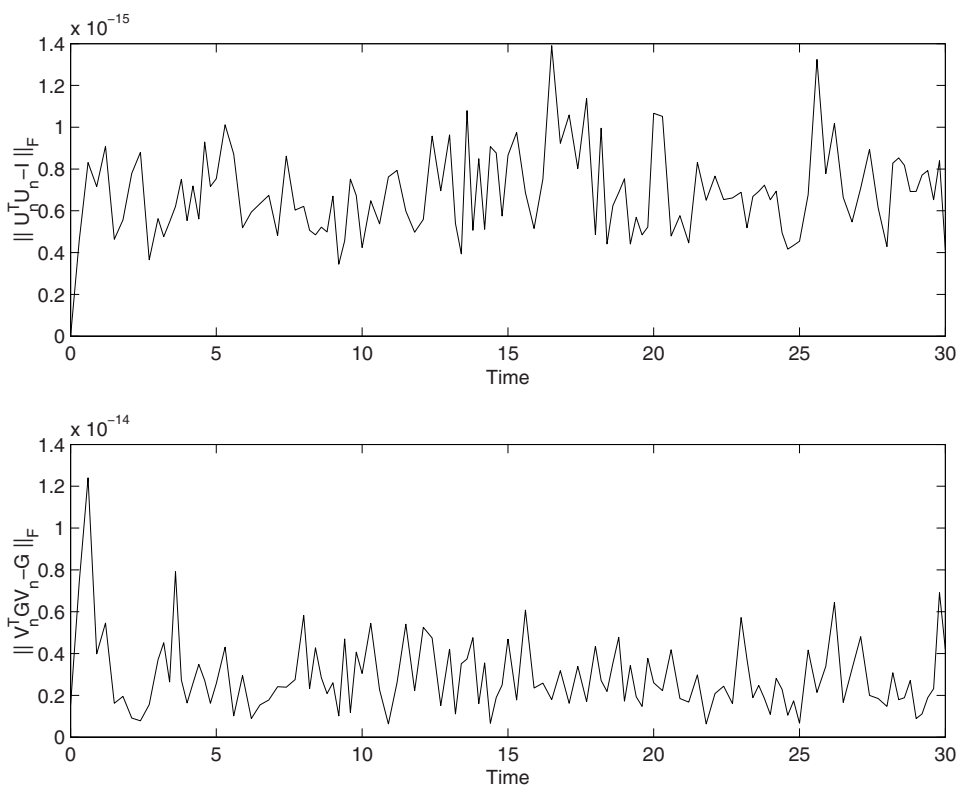

Fig. 3. Errors in the orthogonal and in the quadratic group related to $G$.
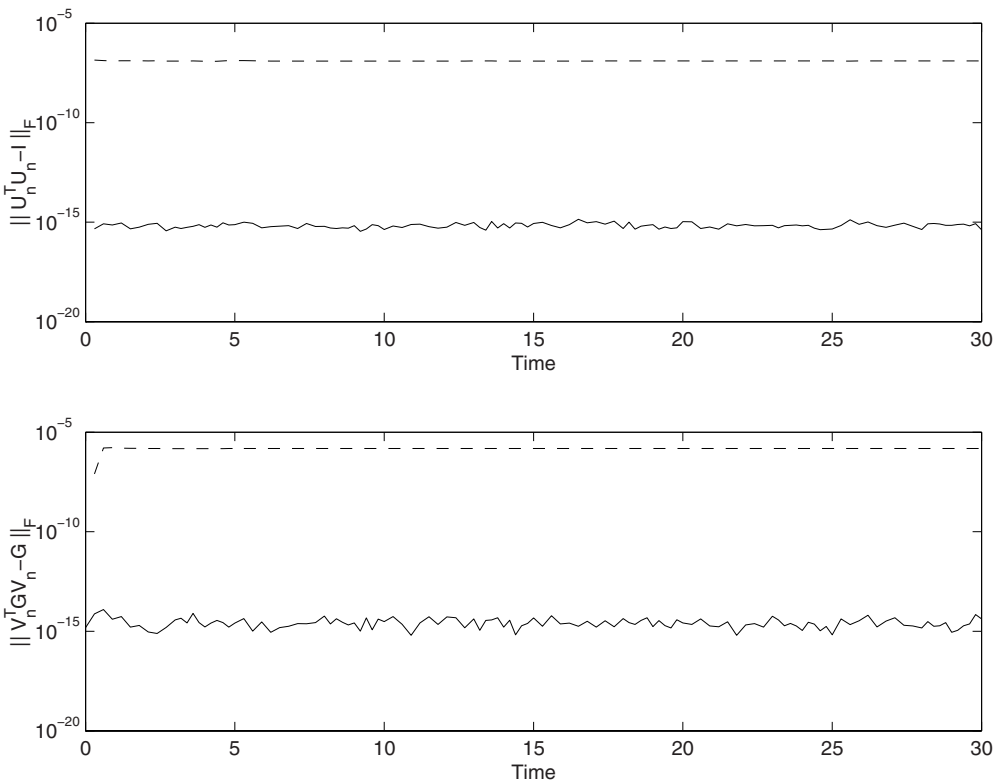

Fig. 4. 
proved that the order of the ODE integrator is preserved. The initial conditions for (6) and (17) are random matrices in the manifolds. We observe that the behaviour of the diagonal elements of $X_{n}$ approaches the theoretical values already when $t \simeq 8$. In Figure 3 we show the departure from the orthogonal manifold and the quadratic group related to $G$ for $U_{n}$ and $V_{n}$ respectively, computed as $\left\|U_{n}^{T} U_{n}-I_{n}\right\|_{F}$ and $\left\|V_{n}^{T} G V_{n}-G\right\|_{F}$. Finally in Figure 4 we show that the use of the projection of the numerical solution on the manifolds is necessary. In the picture the solid lines denote the manifold errors of the numerical solutions for $U(t)$ and $V(t)$ using the projection while the dashed lines denote the errors given by the MatLab integrator. The solution is computed by routine ode113 with a relative tolerance set to $10^{-6}$ but it departes from the manifold very soon.

\section{References}

1. Bojanczyk A.W., Onn R., Steinhardt A.O.: Existence of the Hyperbolic Singular Value Decomposition. Lin. Alg. Appl. 185 (1993) 21-30

2. Chu M.T., Driessel K.R.: The projected gradient method for least square matrix approximations with spectral constraints. SIAM J. Numer. Anal. 27 (1990) 10501060

3. Dieci L., Russell R.D., Van Vleck E.S.: Unitary integrators and applications to continuous orthonormalization techniques. SIAM J. Numer. Anal. 31 (1994) 261281

4. Diele F., Lopez L., Peluso R.: The Cayley transform in the numerical solution of unitary differential systems. Adv. Comput. Math. 8 (1998) 317-334

5. Higham N.: J-Orthogonal matrices: properties and generation. SIAM Rev. 45 (3) (2003) 504-519

6. Iserles A., Munthe-Kaas H., Nørsett S.P., Zanna A.: Lie-group methods. Acta Numerica 9 (2000) 215-365

7. Lopez L., Politi T.: Applications of the Cayley approach in the numerical solution of matrix differential systems on quadratic groups. Appl. Num. Math. 36 (2001) $35-55$

8. Onn R., Steinhardt A.O., Bojanczyk A.W.: The Hyperbolic Singular Value Decomposition and Applications. IEEE Trans. Sign. Proc. 39 (7) (1991) 1575-1588

9. Slapničar I.: Componentwise analysis of direct factorization of real symmetric and Hermitian matrices. Lin. Alg. Appl. 272 (1998) 227-275

10. Slapničar I.: Highly accurate symmetric eigenvalue decomposition and Hyperbolic SVD. Lin. Alg. Appl. 358 (2003) 387-424

11. Stewart M., Stewart G.W.: On hyperbolic triangularization: stability and pivoting. SIAM J. Matrix Anal. Appl. 19 (4) (1998) 847-860.

12. Veselić K.: A Jacobi eigenreduction algorithm for definite matrix pairs. Numer. Math. 64 (1993) 241-269

13. Wright K.: Differential equations for the analytic singular value decomposition of a matrix. Numer. Math. 63 (1992) 283-295 